\title{
Zooming in on virus surface protein mobility
}

\author{
Jakub Chojnacki*,1 \& Christian Eggeling ${ }^{1,2,3}$ \\ ${ }^{1}$ MRC Human Immunology Unit, Weatherall Institute of Molecular Medicine, University of Oxford, Oxford OX3 9DS, UK \\ ${ }^{2}$ Institute of Applied Optics, Faculty of Physics \& Astronomy, Friedrich-Schiller-University Jena, Max-Wien Platz 4, 07743 Jena, \\ Germany \\ ${ }^{3}$ Leibniz Institute of Photonic Technology eV, Department of Biophysical Imaging, Albert-Einstein-Straße 9, 07745 Jena, Germany \\ *Author for correspondence: jakubcho@gmail.com
}

\section{'s successful application of SSTED-FCS in virus research opens a way for dynamic analysis of molecular behavior not only for HIV-1 surface but also for all molecular level virus and cell interactions"}

First draft submitted: 12 January 2018; Accepted for publication: 26 January 2018; Published online: 23 February 2018

Keywords: fluorescence correlation spectroscopy • HIV-1 • STED microscopy • super-resolution microscopy

Fluorescence microscopy is a powerful tool in many life science research areas; thanks to its live-cell compatibility and its ability to observe only specifically labeled molecules of interest. However, in the field of virology, this technique has traditionally been of limited use, specifically due to the fundamental resolution limit associated with the diffraction of the visible light. The diffraction limit makes fluorescence microscopes unable to resolve details below approximately $200 \mathrm{~nm}$ in the focal plane and approximately $600 \mathrm{~nm}$ along the optical axis, thus making studies of approximately 100-nm-sized virus particles infeasible. Therefore, for a long time, virus visualization was performed solely via electron microscopy-based methods, which - with its subnanometre resolution - enabled for numerous insights into details of virus structures. On the other hand, electron microscopy-based methods require laborious preparation of biological sample (fixation or freezing) making them unsuitable for the study of dynamic processes of viruses and virus-cell interactions.

However, this situation began to change with the development of super-resolution fluorescence microscopy (SRFM) or nanoscopy in the 1990s [1,2]. These advanced microscopy techniques surpass the diffraction limit of light, giving molecular-scale spatial resolution, and thus, for the first time, opening up the possibility to observe details of subdiffraction-sized viruses with fluorescence microscopy. Currently, there are many different SRFM techniques available, each with its own set of advantages and disadvantages [3-5]. The requirement for the observation of sub-100 nm sized virus structures limited virological studies to the use of SRFM approaches that routinely offered 10-100 nm spatial resolution, such as stimulated emission depletion (STED) microscopy and single molecule switching microscopy-based approaches like photo-activation localization microscopy (PALM) or stochastic optical reconstruction microscopy (STORM).

HIV-1 was one of the earliest biological systems studied by SRFM, and currently SRFM-based HIV-1 studies have already provided numerous ground breaking insights into retroviral replication cycle [6,7]. However, only very recently have these studies began to transition from the observations of fixed samples into live-cell imaging, which now allows for the study of dynamic aspects of the virus structure and virus-cell interactions. This is due to the fact that, to achieve highest spatial resolutions, SRFM techniques usually require long acquisition times thus making live-cell imaging infeasible especially for observation of molecular dynamics in single viruses.

One of the solutions that allows for live-cell studies of single molecules on the surface of sub- $100 \mathrm{~nm}$ objects lies in combining the existing STED SRFM technique, with single-molecule-based spectroscopic tools such as fluorescence correlation spectroscopy (STED-FCS) [8]. This combination allows for the determination of molecular mobility for observation spot sizes below $60 \mathrm{~nm}$ in diameter, and thus for SRFM studies to be conducted with a very high level of temporal resolution on the relevant spatial scales. Moreover, the combination of STED-FCS with fastbeam scanning (scanning STED-FCS, sSTED-FCS) further extends this technique, enabling molecular mobility 
observations at different spatial points simultaneously, and sSTED-FCS experiments on sphingolipids highlighted transient interaction sites in the plasma membrane of living cells [9].

sSTED-FCS was recently utilized to, for the first time, study the details of protein dynamics on the surface of individual HIV-1 particles [10]. This study demonstrated that the mobility of the virus surface envelope glycoprotein (Env) is dependent on the virus maturation status, which, by viral protease-mediated cleavage of the structural polyprotein protein Gag, converts budding HIV-1 from an immature noninfectious into a fully mature infectious form. Specifically, the disassembly of the immature Gag lattice during maturation was found to correspond with increased Env mobility. This increased mobility represents an underlying mechanism for Env protein clustering in mature virus particles that (by an earlier SRFM-based imaging study) was in the sSTED-FCS measurements found to be important for the productive virus entry [11]. Furthermore, comparing studies of the diffusion characteristics of Env as well as GPI-anchored protein and MHC-I between HIV-1 and cellular surfaces revealed that the HIV-1 surface is a very immobile environment where all proteins display a strongly reduced mobility (two orders of magnitude slower compared with the cell plasma membrane). Therefore, this study in addition to disclosing one of the underlying mechanisms behind maturation-dependent Env clustering highlighted novel insights into molecular properties of the subdiffraction sized highly crowded virus envelope.

In a broader context, this successful application of sSTED-FCS in virus research opens a way for dynamic analysis of molecular behavior not only for HIV-1 surface but also for all molecular level virus and cell interactions that occur during other stages of virus replication cycle, for example, during virus assembly or entry. Moreover, such application of sSTED-FCS is not only limited to HIV-1 or other viruses but can equally be applied to the analysis of small cellular organelles and vesicles such as endosomes or peroxisomes, as well as receptor clustering events that occur during cell-cell interactions such as formation of immunological or virological synapses. Similar to HIV-1, where the maturation-driven mobility changes underpin the ability for the virus to fuse with the target cell, SRFM-based molecular mobility studies may reveal a crucial dependence on a specific dynamic behavior in interacting viral and/or cellular molecules. This knowledge, in turn, may be then used as a source for novel therapies that rather than blocking interacting molecules subtly affect their mobility and consequently inhibit the parts of virus replication cycles such as cell-to-cell transmission or entry.

However, while the above study offers an interesting glimpse into new avenues for future virology research studies; these are arguably still early days of SRFM-based research especially in terms of live-cell experiments. SRFM techniques, while already widespread thanks to the increasing availability of commercial turn-key systems, have only just began to fulfill their potential to become a standard approach for bioimaging. Challenges such as increasing the imaging depth beyond several hundred nanometers while maintaining the resolution and minimizing phototoxicity, still remain a concern for all future live-cell SRFM studies. In addition, sub-100 nm SRFM techniques often require specialized organic dyes, fluorescence proteins or buffer conditions for imaging. Furthermore, while convenient, fluorescence tagging via antibodies or nanobodies (employed in the HIV-1 sSTED-FCS study [10]) provides only a limited usability for live-cell imaging since it restricts the studies to virus or cell external surfaces only. Fortunately, in case of HIV-1, effective strategies based on fluorescence proteins have already been developed for imaging studies via conventional microscopy and these can be adopted for live-cell SRFM. Organic dye compatible tagging approaches that utilize nonfluorescent tags such as SNAP-/HALO-/CLIP-tag or artificial amino acids and click chemistry also provide SFRM-compatible fluorescence labeling for live-cell SRFM studies of virus replication cycle [12]. Finally, as with every scientific tool, sSTED-FCS also carries limitations such as the inability to observe a small number of proteins on the surface of individual viruses for longer than a few seconds due to photobleaching, future mobility studies should also aim to incorporate complementary approaches for single molecule mobility measurements that are less prone to these limitations, such as interferometric scattering microscopy [13].

In summary, the above SRFM-based study of Env mobility on HIV-1 surfaces represents a promising first step, opening a way for future studies that will shed light on the previously undescribed molecular dynamic properties of subdiffraction-sized objects such as viruses or vesicles.

\section{Open access}

This article is distributed under the terms of the Creative Commons Attribution License 4.0 which permits any use, distribution, and reproduction in any medium, provided the original author(s) and the source are credited. To view a copy of the license, visit http://creativecommons.org/licenses/by/4.0/. 


\section{Financial \& competing interests disclosure}

J Chojnacki and C Eggeling are supported by the MRC (grant number MC_UU_12010/unit programs G0902418 and MC_UU_12025), MRC/BBSRC/EPSRC (grant MR/K01577X/1), Wellcome Trust (grant 104924/14/Z/14 and Strategic Award 091911 (Micron)), Deutsche Forschungsgemeinschaft (Research unit 1905 'Structure and function of the peroxisomal translocon') and Oxford internal funds (EPA Cephalosporin Fund and John Fell Fund). The authors have no other relevant affiliations or financial involvement with any organization or entity with a financial interest in or financial conflict with the subject matter or materials discussed in the manuscript apart from those disclosed.

No writing assistance was utilized in the production of this manuscript.

\section{References}

1. Hell SW, Wichmann J. Breaking the diffraction resolution limit by stimulated emission: stimulated-emission-depletion fluorescence microscopy. Opt. Lett. 19(11), 780-782 (1994).

2. Hell SW, Kroug M. Ground-state-depletion fluorscence microscopy: a concept for breaking the diffraction resolution limit. Appl. Phys. B Lasers Opt. 60(5), 495-497 (1995).

3. Sahl SJ, Hell SW, Jakobs S. Fluorescence nanoscopy in cell biology. Nat. Rev. Mol. Cell Biol. 18(11), 685-701 (2017).

4. Eggeling C, Willig KI, Sahl SJ, Hell SW. Lens-based fluorescence nanoscopy. Q. Rev. Biophys. 48(2), 178-243 (2015).

5. Hell SW, Sahl SJ, Bates M et al. The 2015 super-resolution microscopy roadmap. J. Phys. Appl. Phys. 48(44), 443001 (2015).

6. Hanne J, Zila V, Heilemann M, Müller B, Kräusslich H-G. Super-resolved insights into human immunodeficiency virus biology. FEBS Lett. 590(13), 1858-1876 (2016).

7. Chojnacki J, Eggeling C. Super-resolution fluorescence microscopy studies of human immunodeficiency virus. Retrovirology 15 (2018).

8. Eggeling C, Ringemann C, Medda R et al. Direct observation of the nanoscale dynamics of membrane lipids in a living cell. Nature 457(7233), 1159-1162 (2009).

9. Honigmann A, Mueller V, Ta H et al. Scanning STED-FCS reveals spatiotemporal heterogeneity of lipid interaction in the plasma membrane of living cells. Nat. Commun. 5, 5412 (2014).

10. Chojnacki J, Waithe D, Carravilla P et al. Envelope glycoprotein mobility on HIV-1 particles depends on the virus maturation state. Nat. Commun. 8, 545 (2017).

11. Chojnacki J, Staudt T, Glass B et al. Maturation-dependent HIV-1 surface protein redistribution revealed by fluorescence nanoscopy. Science 338(6106), 524-528 (2012).

12. Sakin V, Paci G, Lemke EA, Müller B. Labeling of virus components for advanced, quantitative imaging analyses. FEBS Lett. 590(13), 1896-1914 (2016).

13. Reina F, Galiani S, Shrestha D et al. Complementary studies of lipid membrane dynamics using iSCAT and STED microscopy. Biorxiv doi:10.1101/235564 (2017) (Epub ahead of print). 
